\title{
Successful treatment of an enterovesical fistula due to Crohn's disease with stem cell transplantation:
}

\section{a case report}

\author{
Andrzej Moniuszko ${ }^{1}$, Anna Sarnowska², Wojciech Rogowski ${ }^{3}$, Marek Durlik $^{4}$, Anna Wluka², Grażyna Rydzewska ${ }^{1,5}$ \\ ${ }^{1}$ Department of Gastroenterology with the Inflammatory Bowel Disease Subdivision, Central Clinical Hospital of the Ministry \\ of the Interior and Administration, Warsaw, Poland \\ ${ }^{2}$ Translative Platform for Regenerative Medicine, Polish Academy of Sciences - Mossakowski Medical Research Centre, \\ Warsaw, Poland \\ ${ }^{3}$ Department of Urology and Oncological Urology, Central Clinical Hospital of the Ministry of the Interior and Administration, \\ Warsaw, Poland \\ ${ }^{4}$ Department of Gastroenterology and Transplant Surgery, Central Clinical Hospital of the Ministry of the Interior and \\ Administration, Warsaw, Poland \\ ${ }^{5}$ Faculty of Medicine and Health Science, Jan Kochanowski University, Kielce, Poland
}

Gastroenterology Rev 2018; 13 (4): 332-336

DOI: https://doi.org/10.5114/pg.2018.79814

Address for correspondence: Prof. Grażyna Rydzewska, MD PhD, Andrzej Moniuszko MD PhD, Department of Gastroenterology with the Inflammatory Bowel Disease Subdivision, Central Clinical Hospital of the Ministry of the Interior and Administration, 137 Woloska St, 02-507 Warsaw, Poland, phone: +48 22508 12 40, e-mail: grazyna.rydzewska@cskmswia.pl, moniuszko.a@gmail.com

Crohn's disease (CD) is a chronic, progressive inflammatory disease affecting the digestive tract. Up to $30 \%$ of patients develop a fistulising disease during their lifetime, leading to the development of a variety of fistulas between the gastrointestinal tract and nearby tissues, such as enterocutaneous, enterovesical or enterovaginal fistulas. Even in the era of biological agents, including anti-TNF drugs, approximately one-third of patients will suffer from fistulas that are chronic and resistant to standard treatments [1], which may lead to dangerous and life-threatening complications.

Recently, mesenchymal stem cells (MSC) were tested for the treatment of fistulising disease in phase II and phase III clinical trials [2-5]. Thanks to their high anti-inflammatory and regenerative potential, MSC exerted strong paracrine effects in perianal fistulas, producing positive effects [5]. However, in these trials, the regenerative cells were obtained from healthy donors. Among the various methods used to obtain stromal cells, the isolation of autologous adipose tissue-derived regenerative cells (ADRC) using the Celution system is one of the most novel and clinically applicable. Herein, we provide the first report of complete enterovesical fistula resolution following treatment with autologous ADRC.

A 52-year-old female who had been suffering from CD for 12 years was admitted to our clinic with a severe bacterial urinary tract infection. She had never attained clinical remission using steroids, immunosuppressants, anti-TNF agents with a short dosing interval, or alternative treatments such as cyclosporine, tacrolimus, or thalidomide. Consequently, she suffered from numerous complications, which led to a significant number of laparotomies. She had undergone multiple segmental resections of the small intestine and colectomy with ileostomy. Only maintenance therapy with adalimumab once a week with concomitant azathioprine resulted in a relative clinical remission. However, she still suffered from frequent perianal and peristomal fistulas, which led to several surgeries to reposition the stoma.

At the time of admission, the patient was suffering from pyrexia (up to $39^{\circ} \mathrm{C}$ ) and dysuria; she also noted the passage of air and faeces during urination. The general state of the patient was critical because of severe undernutrition (BMI 15) and progressive urinary tract infection. A urine culture revealed severe polybacterial infection with Klebsiella pneumoniae, Escherichia coli ESBL (+), and Pseudomonas fluorescens, which was only susceptible to carbapenems. Despite the administration of targeted antibiotic therapy, the state of the patient continued to worsen. A cystoscopy performed after the oral application of methylene blue showed the presence of an enterovesical fistula, approximately $3 \mathrm{~cm}$ long and 
$1 \mathrm{~cm}$ wide, with active suppuration of intestinal contents into the urinary bladder.

Surgery was considered a high-risk treatment for several reasons. First, the multiple past interventions had led to the formation of multiple adhesions in the abdomen cavity, leading to a so-called 'frozen abdomen', which made surgical intervention impossible. Second, the healing process would have been heavily impaired because of the critical state of the patient, including the high CD activity and cachexia. Finally, even with maintenance therapy using adalimumab once a week with concomitant thiopurines, the patient suffered from the formation of new and re-opening fistulas. Peristomal and perianal fistulas were both active at the time of admission.

In this case, conservative treatment with biologics was not expected to be effective, and broad-spectrum antibiotics did not stop the progression of sepsis. Because of the critical state of the patient, we decided to use an alternative treatment as a 'rescue therapy'.

Based on promising previous reports that show the effectiveness of the local injection of adipose tissue-de- rived regenerative cells (ADRC) into enterocutaneous [2], enterovaginal [3], and perianal fistulas [4, 5], we decided to apply the novel technique in this particular case. After approval was obtained from an independent Ethics Committee, the local injection of regenerative cells into the enterovesical fistula was performed during cystoscopy.

Manual liposuction was performed under general anaesthesia. A 260-ml aliquot of lipoaspirate was immediately transferred to the CellCelution 800 system (Cytori Therapeutics Inc.). After $2 \mathrm{~h}$ of tissue processing, $5 \mathrm{ml}$ of an ADRC suspension was obtained. During cystoscopy, the suspension was injected around the fistula through an $18 \mathrm{G}$ needle, the size routinely used for botulin injections, in $0.5-\mathrm{ml}$ aliquots into the detrusor muscle (Figure 1).

Over the next few days, the signs of severe infection gradually resolved. Treatment with adalimumab and azathioprine was continued. Cystoscopies performed 12 and 22 days after the treatment revealed no active leakage of the methylene blue colorant into the urinary bladder, and to our surprise, the enterove-
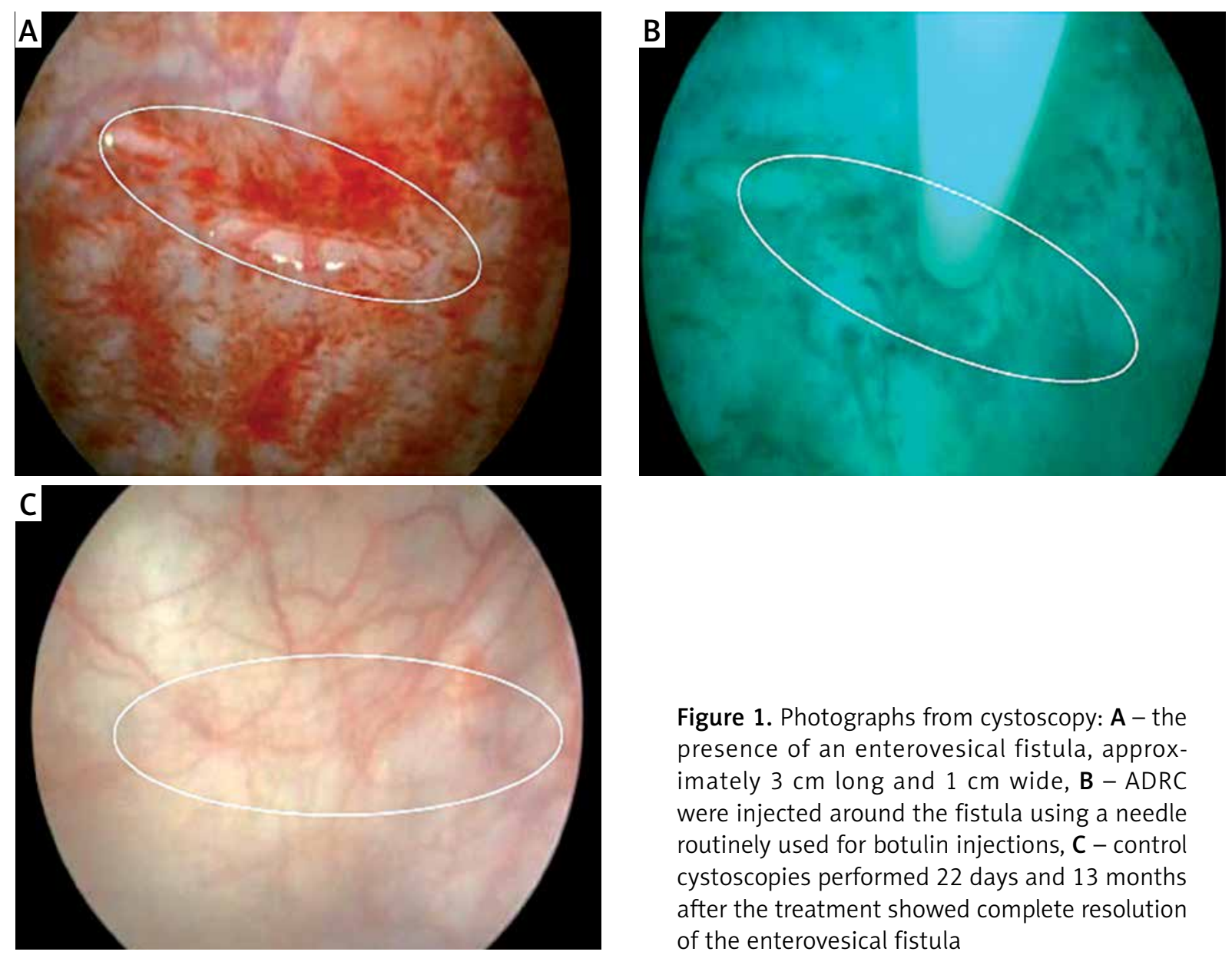

Figure 1. Photographs from cystoscopy: A - the presence of an enterovesical fistula, approximately $3 \mathrm{~cm}$ long and $1 \mathrm{~cm}$ wide, B - ADRC were injected around the fistula using a needle routinely used for botulin injections, $\mathbf{C}$ - control cystoscopies performed 22 days and 13 months after the treatment showed complete resolution of the enterovesical fistula 
sical fistula had completely resolved. Urine culture did not reveal any evidence of pathogenic bacteria. No adverse events occurred during hospitalisation or during one year of clinical observation. Follow-up cystoscopy performed 13 months after the treatment demonstrated complete fistula healing. The clinical effect was maintained after 36 months of follow-up, despite subsequent exacerbations of CD leading to escalation of biological treatment.

Using a 1-ml suspension of ADRC, the phenotype and expression of molecular markers were evaluated using microscopy and fluorescence-activated cell-sort-

\section{A}
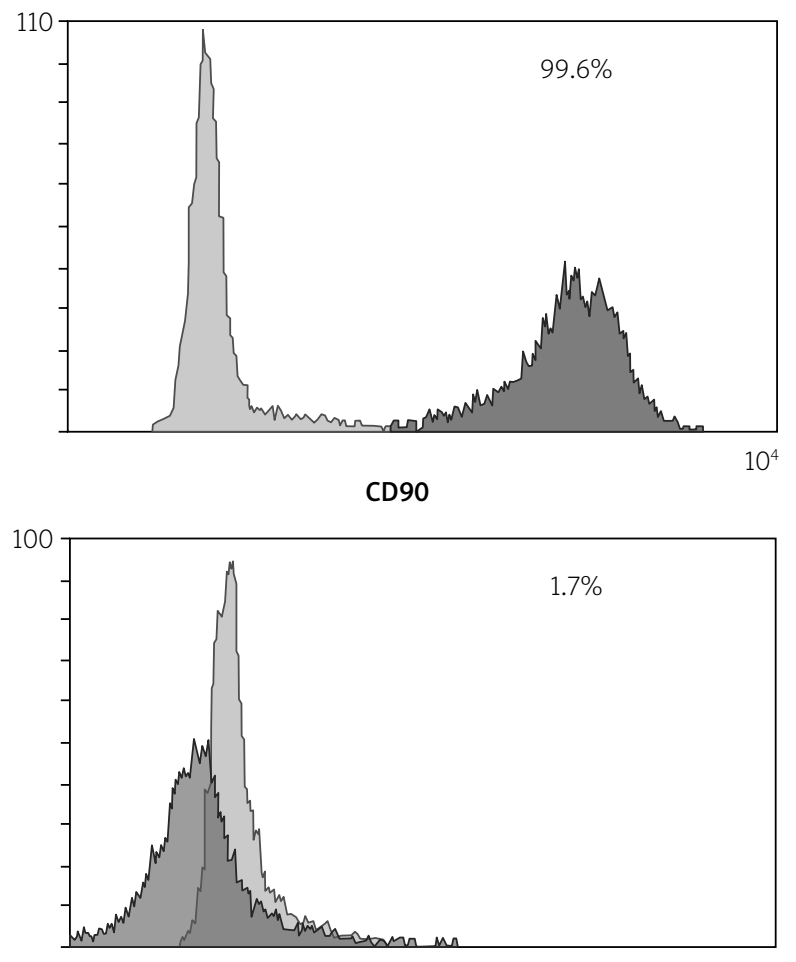

CD34, CD11B, CD19, CD45, HLA-DR

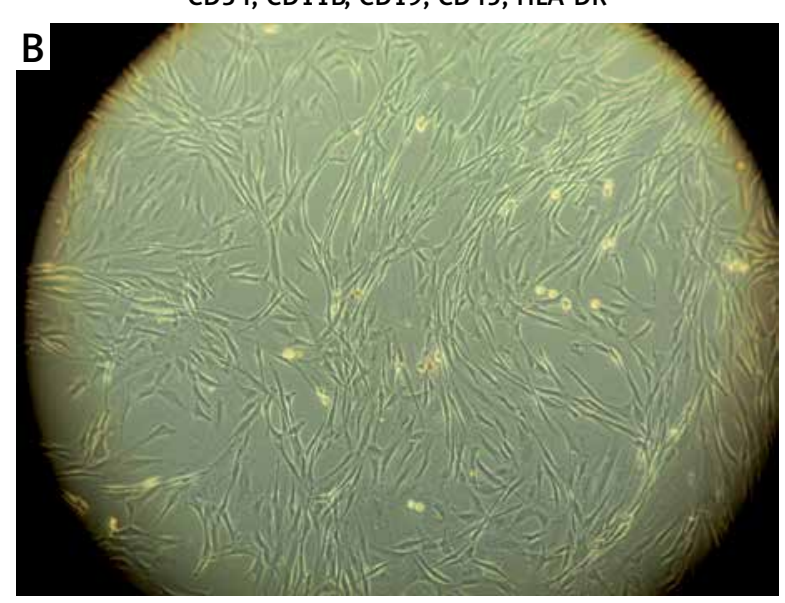

ing (FACS). Freshly isolated cells were counted $(27 \times$ $10^{6} / 5 \mathrm{ml} \mathrm{ADRC}$ ), and cells with $95 \%$ viability were obtained. The cell suspension was cultured in a standard humidified incubator under a $21 \%-\mathrm{O}_{2}$ and $5 \%-\mathrm{CO}_{2}$ atmosphere at $37^{\circ} \mathrm{C}$. After several days, the ADRC became spindle-shaped and fibre-like. The obtained ADRC expressed CD73 (99.3\% of cells), CD90 (99.6\%), and CD105 (89.1\%) as well as CD34, CD19, CD11b, and HLADR (all in $1.7 \%$ of cells) as surface markers (Figure 2).

The evolution of lesions in CD has a significant inflammation-induced component. Unfortunately, the currently used immunosuppressive therapy is burdensome
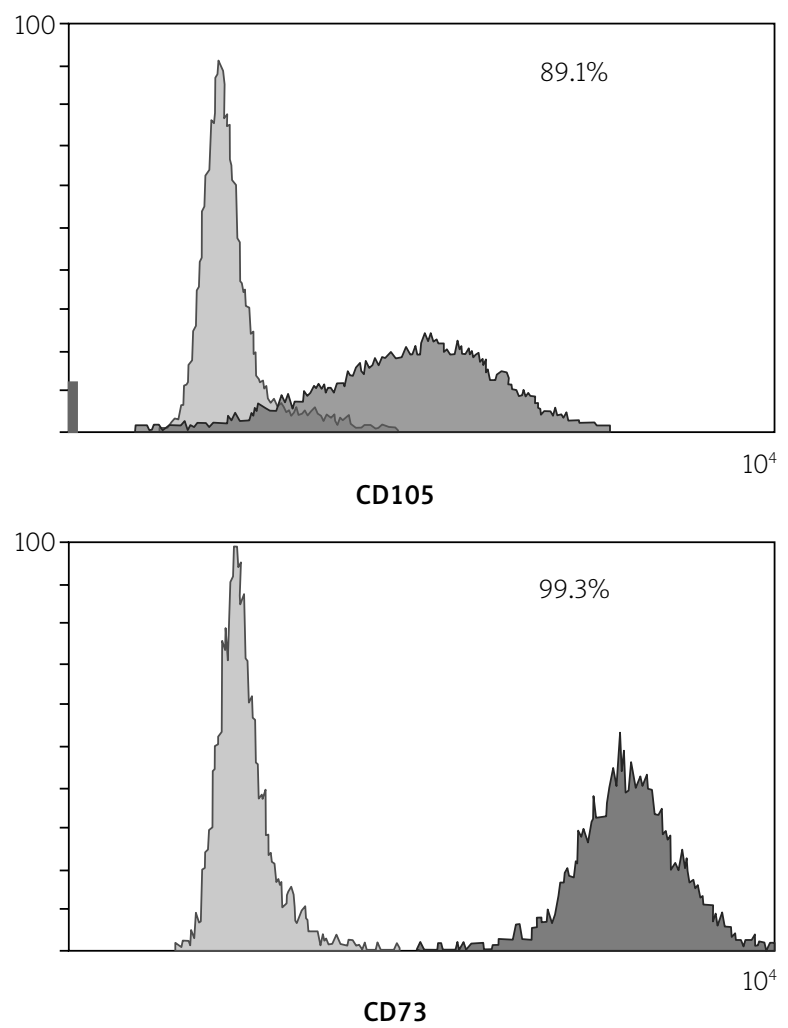

Figure 2. The acquisition of an MSC phenotype by the ADRC (freshly isolated). A - Typical CDMSC markers as determined by FACS, B - the ADRC phenotype in standard culture 
for the patient and not always effective [1]. Thus, the application of ADRC is thought to be a promising therapy. ADRCs have been described to modulate and reduce the inflammatory response through paracrine effects [6]. So far, the application of these cells in CD has been described in only a few papers and has been mainly used in the treatment of enterovaginal and enterocutaneous fistulas $[7,8]$. However, only their effectiveness in healing perianal fistulas has been assessed in large randomised clinical trials, mainly using allogenic adipose-derived MSC. There have been no previous case reports concerning the use of ADRC to treat enterovesical fistulas.

In the phase I/Ila clinical trial concerning persistent fistulas in CD conducted by de la Portilla et al. [5], the authors demonstrated a significant positive effect of allogenic adipose-derived stem cell-based therapy for complex perianal fistulas. More than half $(56.3 \%)$ of the patients achieved complete closure of the treated fistula. However, the treatment involved long laboratory procedures, e.g. digestion of the healthy donors' adipose tissue, culture and incubation, followed by expansion in vitro and long cryopreservation until use. In a South Korean phase II study [9], 78.8\% of the CD patients suffering from perianal fistulas showed complete closure after the first injection, which was notably superior compared to other studies, possibly due to the higher number of cells used per fistula tract.

In our case, we used freshly isolated autologous cells, not expanded ADRC, and injected them $2 \mathrm{~h}$ after isolation. For the first time, we observed successful closure of an enterovesical fistula in a critically ill patient suffering from CD with a high risk of complications and failure of standard surgical treatment. Adapting the Cytori $^{\odot}$ system reduced the time between the isolation of the adipose tissue and the injection of cells in the operating room. The number of cells obtained and injected around the fistula was comparable to that used in the described clinical trials. We successfully demonstrated the therapeutic effects of ADRC in uroepithelial regeneration and observed no adverse effects.

The mechanisms involved in the closure of an enterovesical fistula might be associated with the immunomodulatory effects of the injected ADRC or with their regenerative potential. Increased regeneration of epithelial integrity modulated by adipose-derived MSC has been shown in an animal model by Chang et al. [10]. The regeneration of the intestinal epithelium, together with a reduction of the inflammatory response, led to increased survival in rats that underwent high-dose abdominal irradiation. Moreover, ADRCs were previously described to have a high potential for differentiation into connective tissue cells [11], which may have con- tributed to the successful closure of the fistula in our patient.

Furthermore, Shi et al. [12] recently documented the differentiation of human adipose-derived stem cells into urothelial cells. In an earlier paper by Jack et al. [13], it was shown that human processed lipoaspirate cells injected into the urinary tract exhibited morphological and phenotypic evidence of smooth muscle incorporation and differentiation over time. In addition, the cells were maintained in the muscle for at least 12 weeks in mice.

As described above, in pre-clinical and clinical studies, different populations of mesenchymal cells derived from adipose tissue were applied. Here, the cells were not characterised prior to administration to the patient. An aliquot of the obtained mesenchymal cells was sent to the research laboratory for a full phenotypic and survival analysis.

In conclusion, it appears that using freshly isolated, autologous ADRC-based therapy could be used as a safe, effective and non-invasive alternative for the treatment of CD patients with enterovesical fistulas, with potential long-term clinical effect. Further research is needed to evaluate the effectiveness and safety of this novel method in IBD patients.

\section{Acknowlegdments}

The study was reviewed and approved by the Ethical Committee of the Central Clinical Hospital of the Ministry of the Interior and Administration, Warsaw, Poland.

\section{Conflict of interest}

The authors declare no conflict of interest.

\section{References}

1. Bougen G, Siproudhis L, Gizard E, et al. Long-term outcome of perianal fistulizing Crohn's disease treated with infliximab. Clin Gastroenterol Hepatol 2013; 11: 975-81.

2. Herreros MD, Garcia-Arranz M, Guadalajara H, et al. Autologous expanded adipose-derived stem cells for the treatment of complex cryptoglandular perianal fistulas: a phase III randomized clinical trial (FATT 1: fistula Advanced Therapy Trial 1) and long-term evaluation. Dis Colon Rectum 2012; 55: 762-72.

3. Garcia-Olmo D, Herreros D, Pascual I, et al. Expanded adipose-derived stem cells for the treatment of complex perianal fistula: a phase II clinical trial. Dis Colon Rectum 2009; 52: 79-86.

4. Guadalajara H, Herreros D, De-La-Quintana P, et al. Long-term follow-up of patients undergoing adipose-derived adult stem cell administration to treat complex perianal fistulas. Int I Colorectal Dis 2012; 27: 595-600.

5. de la Portilla F, Alba F, García-Olmo D, et al. Expanded allogeneic adipose-derived stem cells (eASCs) for the treatment of complex perianal fistula in Crohn's disease: results from 
a multicenter phase I/Ila clinical trial. Int J Colorectal Dis 2013; 28: 313-23.

6. Lysak D, Vlas T, Holubova M, et al. In vitro testing of immunosupressive effects of mesenchymal stromal cells on lymphocytes stimulated with alloantigens. Biomed Pap Med Fac Univ Palacky Olomouc Czech Repub 2013; 157: XX.

7. Garcia-Olmo D, Herreros D, Pascual M, et al. Treatment of enterocutaneous fistula in Crohn's disease with adipose-derived stem cells: a comparison of protocols with and without cell expansion. Int J Colorectal Dis 2009; 24: 27-30.

8. Garcia-Olmo D, Herreros D, De-La-Quintana P, et al. Adipose-derived stem cells in Crohn's rectovaginal fistula. Case Report Med 2010; 2010: 961758.

9. Lee WY, Park KJ, Cho YB, et al. Autologous adipose tissue-derived stem cells treatment demonstrated favorable and sustainable therapeutic effect for Crohn's fistula. Stem Cells 2013; 31: 2575-81.

10. Chang P, Qu Y, Liu Y, et al. Multi-therapeutic effects of human adipose-derived mesenchymal stem cells on radiation-induced intestinal injury. Cell Death Dis 2013; 4: e685.

11. Kang BJ, Lee SH, Kweon OK, et al. Differentiation of canine adipose tissue-derived mesenchymal stem cells towards endothelial progenitor cells. Am J Vet Res 2014; 75: 685-91.

12. Shi JG, Fu WJ, Wang XX, et al. Transdifferentiation of human adipose-derived stem cells into urothelial cells: potential for urinary tract tissue engineering. Cell Tissue Res 2012; 347: 737-46.

13. Jack GS, Almeida FG, Zhang R, et al. Processed lipoaspirate cells for tissue engineering of the lower urinary tract: implications for the treatment of stress urinary incontinence and bladder reconstruction. J Urol 2005; 174: 2041-5.

Received: 8.10.2018

Accepted: 10.10 .2018 